\title{
Brain connectivity in pathological and pharmacological coma
}

\author{
Quentin Noirhomme ${ }^{1 * t}$, Andrea Soddu ${ }^{1+}$, Rémy Lehembre ${ }^{1,2}$, Audrey Vanhaudenhuyse ${ }^{1}$, Pierre Boveroux ${ }^{1,3}$, \\ Mélanie Boly ${ }^{1}$ and Steven Laureys ${ }^{1}$
}

1 Coma Science Group, Cyclotron Research Centre and Neurology Department, University and University Hospital of Liège, Liège, Belgium

${ }^{2}$ Communications and Remote Sensing Laboratory, School of Engineering, Université Catholique de Louvain, Louvain-la-Neuve, Belgium

${ }^{3}$ Department of Anesthesiology, University Hospital of Liège, Liège, Belgium

Edited by:

Silvina G. Horovitz, National Institutes of Health, USA

Reviewed by:

Silvina G. Horovitz, National Institutes of Health, USA

Allen R. Braun, National Institutes of Health, USA

\section{*Correspondence:}

Quentin Noirhomme, Cyclotron

Research Centre, University of Liège,

Bat B30, Allée du 6 août 8, 4000 Liège,

Belgium.

e-mail:quentin.noirhomme@ulg.ac.be

${ }^{\dagger}$ Quentin Noirhomme and Andrea

Soddu contributed equally to this work.
Recent studies in patients with disorders of consciousness (DOC) tend to support the view that awareness is not related to activity in a single brain region but to thalamo-cortical connectivity in the frontoparietal network. Functional neuroimaging studies have shown preserved albeit disconnected low-level cortical activation in response to external stimulation in patients in a "vegetative state" or unresponsive wakefulness syndrome. While activation of these "primary" sensory cortices does not necessarily reflect conscious awareness, activation in higher-order associative cortices in minimally conscious state patients seems to herald some residual perceptual awareness. PET studies have identified a metabolic dysfunction in a widespread frontoparietal "global neuronal workspace" in DOC patients including the midline default mode network ("intrinsic" system) and the lateral frontoparietal cortices or "extrinsic system." Recent studies have investigated the relation of awareness to the functional connectivity within intrinsic and extrinsic networks, and with the thalami in both pathological and pharmacological coma. In brain damaged patients, connectivity in all default network areas was found to be non-linearly correlated with the degree of clinical consciousness impairment, ranging from healthy controls and locked-in syndrome to minimally conscious, vegetative, coma, and brain dead patients. Anesthesia-induced loss of consciousness was also shown to correlate with a global decrease in cortico-cortical and thalamo-cortical connectivity in both intrinsic and extrinsic networks, but not in auditory, or visual networks. In anesthesia, unconsciousness was also associated with a loss of cross-modal interactions between networks. These results suggest that conscious awareness critically depends on the functional integrity of thalamo-cortical and cortico-cortical frontoparietal connectivity within and between "intrinsic" and "extrinsic" brain networks.

Keywords: disorders of consciousness, connectivity, anesthesia, fMRI, PET, coma, default mode network, consciousness

\section{INTRODUCTION}

Functional brain imaging and particularly fMRI and PET is increasingly showing its interest in the diagnosis (Laureys et al., 2000a; Hirsch, 2005; Schiff et al., 2005; Phillips et al., 2010) and prognosis (Di et al., 2008; Coleman et al., 2009) of patients with disorders of consciousness (DOC). Active paradigms are now enabling to probe patients' awareness and communication by identifying command following independent of muscle activity (Monti et al., 2010). However, besides some exceptional cases, in the absence of a full understanding of the neural correlates of consciousness, even a near-to-normal activation in response to passive stimulation (e.g., auditory, visual, or somatosensory) can be difficult to interpret in terms of unequivocal proofs of consciousness. All that can be inferred is that a specific brain region is, to some degree, still able to activate and process relevant sensory stimuli. Studies on pharmacological coma, i.e., general anesthesia, can help us better understand residual brain functional integration and perception in patients with DOC. Healthy volunteers studied under anesthesia may show residual activation of segregated cortical islands in response to external stimuli (auditory, visual, or somatosensory) encompassing the brainstem, thalamus, and "low-level" primary cortices, similar to findings obtained in unconscious DOC patients.
Higher-order areas encompassing lateral and midline frontoparietal networks show no activation in either volunteers under deep anesthesia or in patients in a "vegetative state." At the opposite, patients in minimally conscious state can show activation of the higher-order areas sometimes similar to healthy control subjects (Laureys, 2005).

A common finding of studies on both pathological and pharmacological coma is an impairment in the activity of a widespread cortical network, encompassing bilateral frontoparietal associative cortices, but not of "lower level" sensory cortices (Baars et al., 2003; Alkire and Miller, 2005; Laureys, 2005). As we will see, a decrease in regional activation seems not sufficient to loose awareness but also requires a functional disconnection within that network and with the thalami, in line with a number of current theoretical views on consciousness (Tononi, 2004; Dehaene et al., 2006; Seth et al., 2006).

\section{ALTERED STATES OF CONSCIOUSNESS}

Altered states of consciousness here refer to an alteration in the level and content of conscious awareness during to sleep, general anesthesia, seizure or coma, and related states. In the latter case, they are coined DOC and encompass a spectrum of clinical conditions 
involving profound disruption in wakefulness and/or awareness due to severe brain lesions (Giacino et al., 2002; Laureys et al., 2004; Bernat, 2006; Schiff, 2006). The clinical definition of consciousness distinguishes between two components, namely wakefulness and awareness (Laureys, 2005). Patients in coma are unconscious because they cannot be awakened (i.e., the never open the eyes). Following coma, some patients may "awaken" (meaning they open the eyes) but remain unaware. This condition is called the "vegetative state" (Jennett and Plum, 1972) recently renamed "unresponsive wakefulness syndrome" (Laureys et al., 2010).

Minimally conscious state refers to patients who are unable to reliably communicate but show reproducible albeit fluctuating behavioral evidence of awareness (i.e., non-reflex movements or command following; Giacino et al., 2002). Locked-in syndrome patients (Plum and Posner, 1972) are fully conscious but are completely paralyzed except for small movements of the eyes or eyelids.

Pharmacologically induced unconscious states are commonly named deep sedation or general anesthesia. All sedative anesthetic agents do not act on the brain in the same way. They can be separated into three main categories: (i) classic anesthetic agents (e.g., propofol, midazolam, halothane, isoflurane, or sevoflurane) are able to induce graded states of sedation and combine alterations of wakefulness and awareness; (ii) dissociative anesthetic agents (e.g., ketamine or nitrous oxide), are able to blunt out conscious processes while maintaining signs of wakefulness; and (iii) minimally sedative agents induce graded alterations of wakefulness while preserving several cognitive brain functions (Boveroux et al., 2008). At low-sedative doses anesthetic agents may cause a state similar to drunkenness and subjects may present an increased sleepiness distorted time perception and depersonalization (Alkire et al., 2008b). Furthermore, they show a reduced response to pain and loss of memory. Next, when the anesthetic dose is increased, the subject will fail to move in response to commands and is therefore considered unconscious. During surgery, higher anesthetic doses are used to prevent movement and response to painful stimulation, also ensuring stable hemodynamic function.

\section{DISCONNECTED ISLANDS OF SENSORY “LOW-LEVEL” CORTICAL ACTIVATION}

In response to external stimulation, islands of activation can be observed in the brain of patients with DOC (Laureys et al., 2004; Laureys, 2005; Boly et al., 2008a; Owen and Coleman, 2008), or anesthetized healthy subjects (Alkire and Miller, 2005; Ramani and Wardhan, 2008). Few studies looked at the functional connectivity between these cortical islands and the rest of the brain. PET $\mathrm{H}_{2} \mathrm{O}^{15}$ activation studies on auditory processing in patients with DOC using psychophysiological interaction analyses (Friston et al., 1997), showed a higher functional connectivity between auditory sensory cortex and a widespread network of "higher-order" frontotemporoparietal areas in normal volunteers (Laureys et al., 2000a, 2002) and in patients in a minimally conscious state (Boly et al., 2004) as compared to patients in a vegetative/unresponsive state. Similar psychophysiological interaction analyses of noxious processing revealed preserved modulation between primary somatosensory cortex and a large set of associative areas, again including frontoparietal associative areas, in patients in minimally conscious state
(Boly et al., 2008a) that was not observed in patients in a vegetative/ unresponsive state (Laureys et al., 2002). Compared with healthy controls, patients in minimally conscious state had impaired connectivity between sensory cortex and anterior and posterior midline cortices (Boly et al., 2008a).

Resting state fMRI studies with pharmacologically induced loss of consciousness have shown contrasting results. In a study using midazolam sedation (Greicius et al., 2008), independent component analysis (McKeown and Sejnowski, 1998) was used to isolate sensory-motor, mid-cingulate, and supplementary motor networks. At low-sedative dose without loss of consciousness, the network was still covering both hemispheres and increased connectivity was found in the mid-cingulate area. These results are in line with an fMRI resting state study (Kiviniemi et al., 2005) employing correlation analyses showing increased correlation at low-sedative dose in "low-level" sensory areas. However, a PET study (White and Alkire, 2003) employing higher sedation dose with either halothane or isoflurane leading to loss of consciousness demonstrated (using psychophysiological interaction analyses) a decreased functional connectivity between the thalamus and primary motor cortex, and between the thalamus and supplemental motor area. In the same study, a structural equation modeling (assessing effective connectivity; McIntosh and Lobaugh, 2004) showed disconnections between supplementary motor and thalamic areas and between the former and primary motor cortex. A fMRI resting state study using sevoflurane anesthesia (again leading to loss of consciousness) and a seed-voxel cross-correlation analysis also showed a decreased connectivity of the primary motor cortex (Peltier et al., 2005). In this study, the activity of the seed region in the primary motor cortex in the awake state correlated with bilateral sensorimotor and supplementary motor areas. When sevoflurane was given upto the point of loss of consciousness, the seed exhibited reduced connectivity with the opposite hemisphere. At higher even doses of the anesthetic, functional connectivity was virtually absent. We recently assessed functional connectivity in auditory and visual networks by means of both independent component and seed-correlation analyses during induced propofol anesthesia using resting state fMRI (Boveroux et al., 2010). When comparing deep sedation to wakefulness, no significant differences in cortico-cortical and thalamo-cortical connectivity could be identified in early visual and auditory networks. On the other hand, we found a linear decrease in cortico-cortical connectivity with the level of sedation of "higher-order" associative areas. Finally, Alkire et al. (2008a), studied the influence of emotion on memory during light sedation with structural equation modeling. During 0.25\% sevoflurane anesthesia (a subanesthetic dose), the connections between right hippocampus and amygdala and between right hippocampus and nucleus basalis of Meynert were suppressed, as compared to normal wakefulness.

\section{“HIGHER-ORDER" FRONTOPARIETAL ACTIVATION}

A large frontoparietal network encompassing bilateral frontal and temporo-parietal associative cortices has its activity commonly impaired during altered states of consciousness (Baars et al., 2003; Alkire and Miller, 2005; Laureys, 2005) (Figure 1). This network can be divided into several parts with distinct functions (Boly et al., 2008b,c; Vanhaudenhuyse et al., 2010a). In particular, a distinction can be made between a network involved in the awareness of 


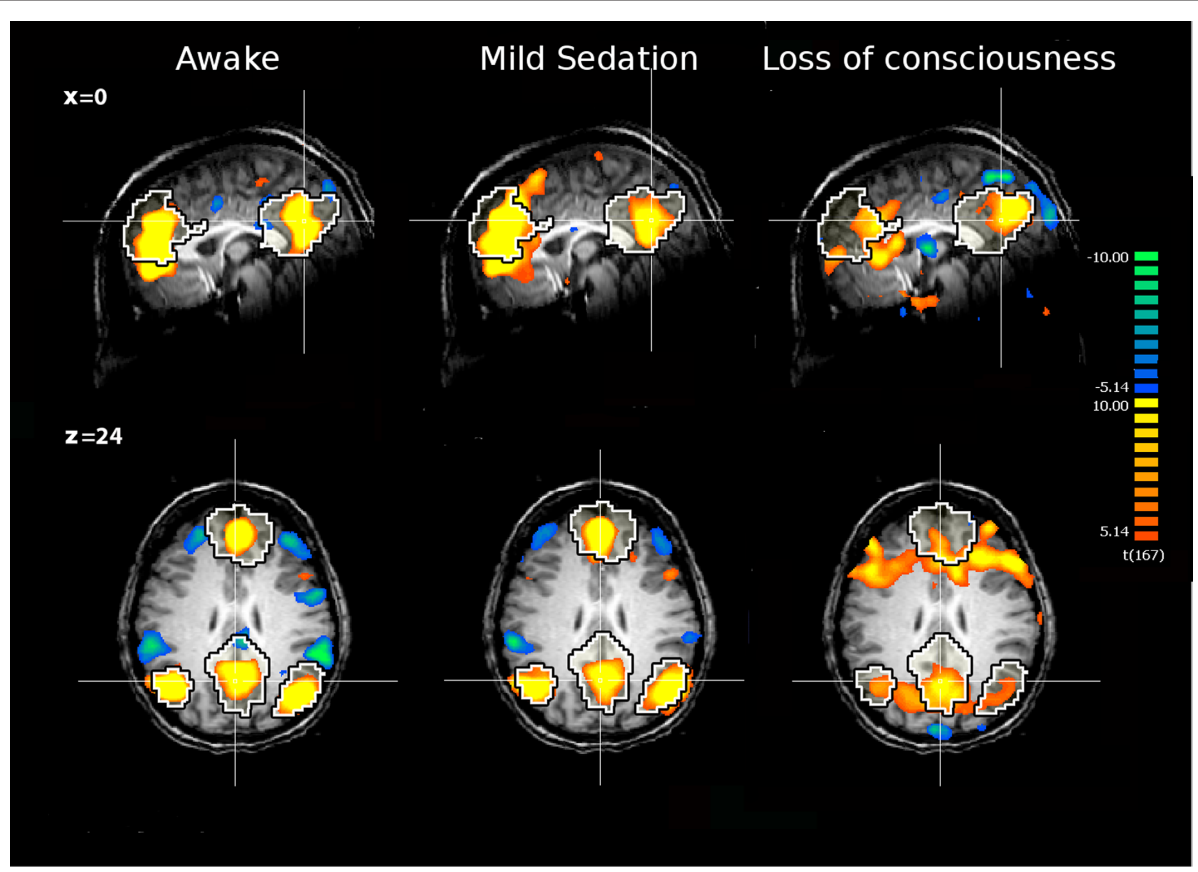

FIGURE 1 |The internal network of a healthy subject awake, under mild sedation and after loss of consciousness. The network was extracted with ICA. The black and white contour represents a template of the internal network extracted from 11 awake healthy subjects with ICA. Yellow and orange colors represent the areas which activities positively correlate with the time course of the internal network. The green and blue colors represent the areas which activities are anticorrelated with activities of the internal network, i.e., the external network. The anticorrelation disappears during deep sedation. The figure is based on data from Boveroux et al. (2010).

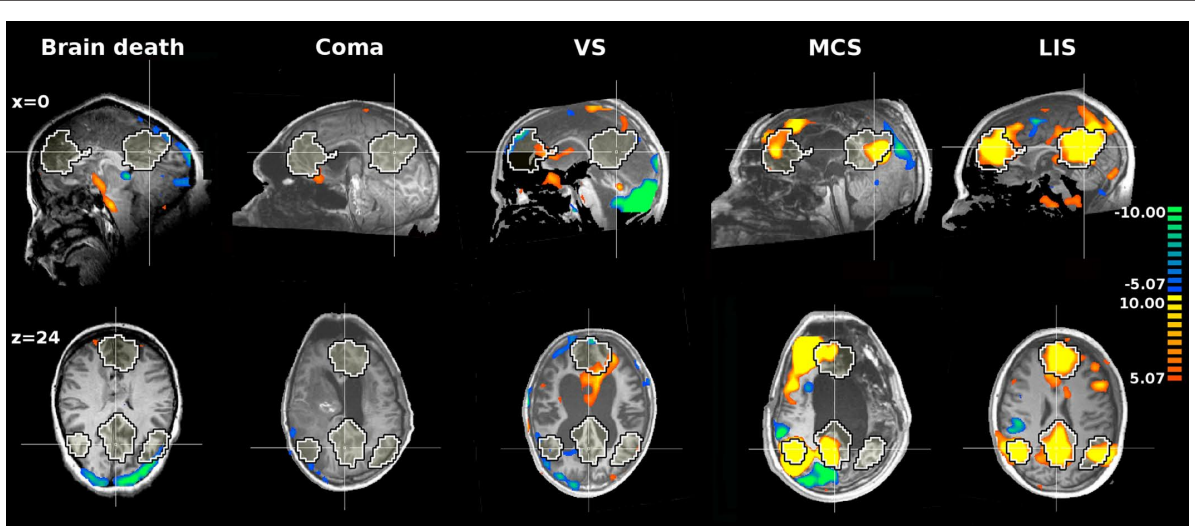

FIGURE 2 |The internal network of patients with brain death, coma, vegetative state (VS), minimally conscious state (MCS), and Locked-in syndrome (LIS). The network was extracted with ICA. The black and white contour represents a template of the internal network extracted from 11 awake healthy subjects with ICA. Yellow and orange colors represent the areas which activities positively correlate with the time course of the internal network. The figure is based on data from Boly et al. (2009) and Vanhaudenhuyse et al. (2010b). self (the "internal” midline default mode network) encompassing precuneus/posterior cingulate, mesas-frontal/anterior cingulate, and temporo-parietal cortices (Gusnard and Raichle, 2001; Mason et al., 2007) and an "external" more lateral and dorsal frontoparietal network involved in the awareness of environment (Boly et al., 2008b). Activities of both internal and external networks are anticorrelated.

Functional connectivity studies with PET and psychophysiological interaction analyses identified a disturbed cortico-cortical connectivity within the frontoparietal network in patients in vegetative/ unresponsive state (Laureys et al., 1999). More recently, fMRI studies on the default/internal network confirmed a decreased corticocortical connectivity in patients with DOC (Boly et al., 2009; Cauda et al., 2009; Vanhaudenhuyse et al., 2010b) and an absence of connectivity in brain death (Boly et al., 2009) (Figure 2). Paralleling clinical experience, a non-linear correlation was found between this default/"internal" network connectivity and the level of consciousness ranging from healthy volunteers and pseudocoma/ locked-in syndrome, to minimally conscious, vegetative/unresponsive, and comatose patients (Vanhaudenhuyse et al., 2010b). 
Furthermore, precuneus connectivity was found to be significantly stronger in minimally conscious patients compared to vegetative/ unresponsive state patients, while locked-in syndrome patients' default/"internal" network connectivity was shown not to be significantly different from healthy control subjects. Finally, thalamocortical connectivity with the higher-order frontoparietal network was only studied in patients in vegetative/unresponsive state with $\mathrm{H}_{2} \mathrm{O}^{15}$ PET (Laureys et al., 2000b) where it shown to be severely disturbed and recovered near normal modulation after recovery of consciousness.

Functional connectivity studies during anesthesia reported decreased cortico-cortical connectivity in "internal” (Greicius et al., 2008; Boveroux et al., 2010) and “external” (Boveroux et al., 2010) networks in healthy volunteers during light sedation with either midazolam or propofol. During deep sedation with propofol inducing clinical unconsciousness, partially preserved residual functional connectivity could be identified both in the internal and external networks (Figure 1). Across sedation states, functional connectivity strength in key nodes of both networks and in thalamo-cortical connectivity with internal and external networks showed a linear correlation with decreasing consciousness (Boveroux et al., 2010). Finally, anticorrelations between internal and external networks also showed a linear trend and could not be identified during loss of consciousness.

\section{FUTURE DIRECTIONS}

In conclusion, PET and fMRI connectivity studies on pathological and pharmacological coma emphasize the two different kinds of brain function that can be encountered during unconsciousness. On one hand, the functional connectivity in "low-level" sensory networks (i.e., auditory, somatosensory, visual, and motor) seems independent of the level and content of consciousness. On the other hand, the connectivity between these primary cortices and "higher-order" associative cortices as well as connectivity inside the nodes of the default/"internal" network show a correlation decreasing consciousness in both coma and anesthesia. Thalamo-cortical connectivity follows the same distinction with preserved connectivity between the thalamus and primary cortices, and impaired connectivity between the thalamus and the default/“internal" and perceptual/"external” networks during states of altered consciousness.

Even when consciousness vanishes, i.e., in vegetative/unresponsive state or anesthesia, residual functional connectivity can be identified in both networks. These results are in line with recent findings in anesthetized monkey (Vincent et al., 2007), and in humans during light and slow wave sleep (Horovitz et al., 2008, 2009). During light sleep, the functional connectivity of the default/"internal" network is partially preserved, while during slow wave sleep, there is a functional disconnection between frontal and parietal nodes of the networks. The preservation of functional connectivity in the absence of consciousness could be seen as reflecting preserved anatomical/structural connections with some degree of basal functional connectivity dissociated from higher cognitive functions as they disappear in brain death (Boly et al., 2009).

Taken together, these findings suggest a two-layer view of resting state fMRI "internal" and "external" network connectivity: one part of the connectivity would persist independently of the level or content of consciousness, and possibly be related to underlying anatomical connectivity (Vincent et al., 2007; Greicius et al., 2009), and the other part being more tightly related to the presence of conscious cognitive processes (Vanhaudenhuyse et al., 2010a,b). fMRI connectivity studies in patients with DOC need to deal with major methodological issues (Soddu et al., 2010). When studying non-collaborative patients, especially patients that show a significantly reduced neuronal activity, the first issue is the possible contamination by artifact and noise sources. Major confounds in fMRI acquisitions and analyses are movement, pulse, and respiration artifacts. Furthermore, patients with pathological alterations of consciousness often suffer from severe diffuse brain injuries leading to extreme brain atrophy and secondary ex-vacuo hydrocephalus (i.e., dilated ventricles) or from major focal lesions resulting in a largely deformed brain. This implies that a spatial normalization procedure is difficult to apply, and the selection of the regions under study becomes difficult and requires visual inspection of an expert eye.

In studies on general anesthesia, a potential bias may be that most studies use relatively low sedation levels. Indeed, most if not all studies use just enough sedative agent to reach unconsciousness. Deeper sedation levels as those used in the operating room probably would result in much more profound functional cerebral disconnections. The use of classical anesthesia as a model for studying human consciousness can also be discussed. Indeed, classical sedative anesthetic agents, as all the ones reviewed in the present paper, decrease both wakefulness and awareness while DOC may present a dissociation between both (e.g., the vegetative or unresponsive syndrome). Dissociative agents have some properties more similar to the latter but also some disadvantages related to the induction of confusion and motor agitation making their use in a scientific setting more difficult. Further studies should investigate brain connectivity changes under these different yet challenging dissociative anesthetic agents such as ketamine or xenon.

From a methodological point of view, most presented studies rely on functional connectivity which measures temporal correlation between cerebral activities in distinct brain areas but cannot infer causality. Effective connectivity methods which can infer causality have been proposed but seldom used in studies on consciousness. They suffer from their higher complexity and from the difficulty to assess causality from the low pass filtered hemodynamic signal in fMRI studies. A more straightforward approach would rely on transcranial magnetic stimulation coupled to electroencephalography (TMS-EEG) to infer effective connectivity from the brain response to a TMS pulse. This perturbation approach has shown promising results in sleep (Massimini et al., 2005) and anesthesia (Ferrarelli et al., 2010). Methods specifically developed to measure consciousness based on the information integration theory (Tononi, 2004) like causal density (Seth, 2005) also used connectivity information but remain difficult to measure (Seth et al., 2008) and have never been applied in these contexts. In our view, the upcoming challenges are to apply effective connectivity approaches to altered states of consciousness aiming to infer causality between brain areas, to integrate hemodynamic and electromagnetic information, and to explain empirical data with theoretical models using specific measures of consciousness. 


\section{ACKNOWLEDGMENT}

Steven Laureys is Senior Research Associate; Andrea Soddu, Mélanie Boly, Audrey Vanhaudenhuyse, and Quentin Noirhomme are Postdoctoral Fellows at the Fonds de la Recherche Scientifique (FRS). Audrey Vanhaudenhuyse was also funded by French Speaking Community Concerted Research Action (ARC 06/11-340).

\section{REFERENCES}

Alkire, M. T., Gruver, R., Miller, J., McReynolds, J. R., Hahn, E. L., and Cahill, L. (2008a). Neuroimaging analysis of an anesthetic gas that blocks human emotional memory. Proc. Natl. Acad. Sci. U.S.A. 105, 1722-1727.

Alkire, M. T., Hudetz,A. G., and Tononi, G. (2008b). Consciousness and anesthesia. Science 322, 876-880.

Alkire, M. T., and Miller, J. (2005). General anesthesia and the neural correlates of consciousness. Prog. Brain Res. 150, 229-244.

Baars, B. J., Ramsoy, T. Z., and Laureys, S. (2003). Brain, conscious experience and the observing self. Trends Neurosci. 26, 671-675.

Bernat, J. L. (2006). Chronic disorders of consciousness. Lancet 367, 1181-1192.

Boly, M., Faymonville, M. E., Peigneux, P., Lambermont, B., Damas, P., Del Fiore, G., Degueldre, C., Franck, G., Luxen, A., Lamy, M., Moonen, G., Maquet, P., and Laureys, S. (2004). Auditory processing in severely brain injured patients: differences between the minimally conscious state and the persistent vegetative state. Arch. Neurol. 61, 233-238.

Boly, M., Faymonville, M. E., Schnakers, C., Peigneux, P., Lambermont, B., Phillips, C., Lancellotti, P., Luxen, A., Lamy, M., Moonen, G., Maquet, P., and Laureys, S. (2008a). Perception of pain in the minimally conscious state with PET activation: an observational study. Lancet Neurol. 7, 1013-1020.

Boly, M.,Phillips, C., Balteau,E.,Schnakers, C., Degueldre, C., Moonen, G., Luxen, A., Peigneux, P., Faymonville, M. E., Maquet, P., and Laureys, S. (2008b). Consciousness and cerebral baseline activity fluctuations. Hum. Brain Mapp. 29, 868-874.

Boly, M., Phillips, C., Tshibanda, L., Vanhaudenhuyse, A., Schabus, M., Dang-Vu, T. T., Moonen, G., Hustinx, R., Maquet, P., and Laureys, S. (2008c). Intrinsic brain activity in altered states of consciousness: how conscious is the default mode of brain function? Ann. N. Y. Acad. Sci. 1129, 119-129.

Boly, M., Tshibanda, L., Vanhaudenhuyse, A., Noirhomme, Q., Schnakers, C., Ledoux, D., Boveroux, P., Garweg, C., Lambermont, B., Phillips, C., Luxen, A., Moonen, G., Bassetti, C., Maquet, P., and Laureys, S. (2009). Functional connectivity in the default network during resting state is preserved in a vegetative but not in a brain dead patient. Hum. Brain Mapp. 30, 2393-2400.

Boveroux, P., Bonhomme, V., Boly, M., Vanhaudenhuyse, A., Maquet, P., and Laureys, S. (2008). Brain function in physiologically, pharmacologically, and pathologically altered states of consciousness. Int. Anesthesiol. Clin. 46, 131-146.

Boveroux, P., Vanhaudenhuyse, A., Bruno, M. A., Noirhomme, Q., Lauwick, S., Luxen, A., Degueldre, C., Plenevaux, A., Schnakers, C., Phillips, C., Brichant, J. F., Bonhomme, V., Maquet, P., Greicius, M. D., Laureys, S., and Boly, M. (2010). Breakdown of within- and between-network resting state functional magnetic resonance imaging connectivity during propofol-induced loss of consciousness. Anesthesiology 113, 1038-1053.

Cauda, F., Micon, B. M., Sacco, K., Duca, S., D’Agata, F., Geminiani, G., and Canavero, S. (2009). Disrupted intrinsic functional connectivity in the vegetative state. J. Neurol. Neurosurg. Psychiatr. 80, 429-431.

Coleman, M. R., Bekinschtein, T., Monti, M. M., Owen, A. M., and Pickard, J. D. (2009). A multimodal approach to the assessment of patients with disorders of consciousness. Prog. Brain Res. 177, 231-248.

Dehaene, S., Changeux, J. P., Naccache, L., Sackur, J., and Sergent, C. (2006). Conscious, preconscious, and subliminal processing: a testable taxonomy. Trends Cogn. Sci. 10, 204-211.

Di, H., Boly, M., Weng, X., Ledoux, D., and Laureys, S. (2008). Neuroimaging activation studies in the vegetative state: predictors of recovery? Clin. Med. 8, 502-507.

Ferrarelli, F., Massimini, M., Sarasso, S., Casali, A., Riedner, B. A., Angelini, G., Tononi, G., and Pearce, R. A. (2010). Breakdown in cortical effective connectivity during midazolam-induced loss of consciousness. Proc. Natl. Acad. Sci. U.S.A. 107, 2681-2686.

Friston, K. J., Buechel, C., Fink, G. R., Morris, J., Rolls, E., and Dolan, R. J. (1997). Psychophysiological and modulatory interactions in neuroimaging. Neuroimage 6, 218-229.

Giacino, J. T., Ashwal, S., Childs, N., Cranford, R., Jennett, B., Katz, D. I.,

This work was supported by European Commission (DISCOS, Mindbridge, DECODER, COST), McDonnell Foundation, Mind Science Foundation, FRS, Reine Elisabeth Medical Foundation and University and University Hospital of Liège. The text reflects solely the views of its authors. The European Commission is not liable for any use that may be made of the information contained therein.

Kelly, J. P., Rosenberg, J. H., Whyte, J., Zafonte, R. D., and Zasler, N. D. (2002) The minimally conscious state: definition and diagnostic criteria. Neurology 58, 349-353.

Greicius, M. D., Kiviniemi, V., Tervonen, O., Vainionpaa, V., Alahuhta, S., Reiss, A. L., and Menon, V. (2008). Persistent default-mode network connectivity during light sedation. Hum. Brain Mapp. 29, 839-847.

Greicius, M. D., Supekar, K., Menon, V. and Dougherty, R. F. (2009). Restingstate functional connectivity reflects structural connectivity in the default mode network. Cereb Cortex 19 , 72-78.

Gusnard, D. A., and Raichle, M.E. (2001). Searching for a baseline: functional imaging and the resting human brain. Nat. Rev. Neurosci. 2, 685-694.

Hirsch, J. (2005). Functional neuroimaging during altered states of consciousness: how and what do we measure? Prog. Brain Res. 150, 25-43.

Horovitz, S. G., Braun, A. R., Carr, W. S., Picchioni, D., Balkin, T. J., Fukunaga, M., and Duyn, J.H.(2009). Decoupling of the brain's default mode network during deep sleep. Proc. Natl. Acad. Sci. U.S.A. 106, 11376-11381.

Horovitz, S. G., Fukunaga, M., de Zwart, J. A., van Gelderen, P., Fulton, S. C., Balkin, T. J., and Duyn, J. H. (2008) Low frequency BOLD fluctuations during resting wakefulness and light sleep: a simultaneous EEG-fMRI study. Hum. Brain Mapp. 29, 671-682.

Jennett, B., and Plum, F. (1972). Persistent vegetative state after brain damage. Lancet 299, 734-737. (RN 35:ICU1-4).

Kiviniemi, V. J., Haanpaa, H., Kantola, J. H., Jauhiainen, J., Vainionpaa, V., Alahuhta, S., and Tervonen, O. (2005). Midazolam sedation increases fluctuation and synchrony of the resting brain BOLD signal. Magn. Reson. Imaging 23, 531-537.

Laureys, S. (2005). The neural correlate of (un)awareness: lessons from the vegetative state. Trends Cogn. Sci. 9 556-559.

Laureys, S., Celesia, G. G., Cohadon, F. Lavrijsen, J., León-Carrión, J., Sannita, W. G., Sazbon, L., Schmutzhard, E., von Wild, K. R., Zeman, A., and Dolce, G.; Consciousness tETFoDo. (2010). Unresponsive wakefulness syndrome: a new name for the vegetative state or apallic syndrome. BMC Med. 8, 68 . doi: 10.1186/1741-7015-8-68

Laureys, S., Faymonville, M. E., Degueldre, C., Fiore, G. D., Damas, P., Lambermont, B., Janssens, N., Aerts, J., Franck, G., Luxen, A., Moonen, G., Lamy, M., and Maquet, P. (2000a). Auditory processing in the vegetative state. Brain 123(Pt 8), 1589-1601.

Laureys, S., Faymonville, M. E., Luxen, A., Lamy, M., Franck, G., and Maquet, P. (2000b). Restoration of thalamocortical connectivity after recovery from persistent vegetative state. Lancet 355 , 1790-1791.

Laureys, S., Faymonville, M. E., Peigneux, P., Damas, P., Lambermont, B., Del Fiore, G., Degueldre, C., Aerts, J., Luxen, A., Franck, G., Lamy, M., Moonen, G., and Maquet, P. (2002). Cortical processing of noxious somatosensory stimuli in the persistent vegetative state. Neuroimage 17, 732-741.

Laureys, S., Goldman, S., Phillips, C., Van Bogaert, P., Aerts, J., Luxen, A., Franck, G., and Maquet, P. (1999). Impaired effective cortical connectivity in vegetative state: preliminary investigation using PET. Neuroimage 9, 377-382.

Laureys, S., Owen, A. M., and Schiff, N. D. (2004). Brain function in coma, vegetative state, and related disorders. Lancet Neurol. 3, 537-546.

Mason, M. F., Norton, M. I., Van Horn, J. D., Wegner, D. M., Grafton, S. T., and Macrae, C. N. (2007). Wandering minds: the default network and stimulus-independent thought. Science 315, 393-395.

Massimini, M., Ferrarelli, F., Huber, R., Esser, S. K., Singh, H., and Tononi, G. (2005). Breakdown of cortical effective connectivity during sleep. Science 309, 2228-2232.

McIntosh, A. R., and Lobaugh, N. J. (2004). Partial least squares analysis of neuroimaging data: applications and advances. Neuroimage 23(Suppl. 1), S250-S263.

McKeown, M. J., and Sejnowski, T.J. (1998). Independent component analysis of fMRI data: examining the assumptions. Hum. Brain Mapp. 6, 368-372.

Monti, M. M., Vanhaudenhuyse, A., Coleman, M. R., Boly, M., Pickard, J. D., Tshibanda, L., Owen, A. M., and Laureys, S. (2010). Willful modulation of brain activity in disorders of consciousness. N. Engl. J. Med. 362, 579-589. 
Owen, A. M., and Coleman, M. R. (2008). Functional neuroimaging of the vegetative state. Nat. Rev. Neurosci. 9, 235-243.

Peltier, S. J., Kerssens, C., Hamann, S. B., Sebel, P.S., Byas-Smith, M., and Hu, X.P. (2005). Functional connectivity changes with concentration of sevoflurane anesthesia. Neuroreport 16, 285-288.

Phillips, C. L., Bruno, M. A., Maquet, P., Boly, M., Noirhomme, Q., Schnakers, C., Vanhaudenhuyse, A., Bonjean, M., Hustinx, R., Moonen, G., Luxen, A., and Laureys, S. (2010). "Relevance vector machine" consciousness classifier applied to cerebral metabolism of vegetative and locked-in patients. Neuroimage. (in press).

Plum, F., and Posner, J. B. (1972). The diagnosis of stupor and coma. Contemp. Neurol. Ser. 10, 1-286.

Ramani, R., and Wardhan, R. (2008). Understanding anesthesia through functional imaging. Curr. Opin. Anaesthesiol. 21, 530-536.

Schiff, N.D. (2006). Multimodal neuroimaging approaches to disorders of consciousness. J. Head Trauma Rehabil. 21, 388-397.
Schiff, N. D., Rodriguez-Moreno, D., Kamal, A., Kim, K. H., Giacino, J. T., Plum, F., and Hirsch, J. (2005). fMRI reveals large-scale network activation in minimally conscious patients. Neurology 64, 514-523.

Seth, A. K. (2005). Causal connectivity of evolved neural networks during behavior. Network 16, 35-54.

Seth, A. K., Dienes, Z., Cleeremans, A., Overgaard, M., and Pessoa, L. (2008). Measuring consciousness: relating behavioural and neurophysiological approaches. Trends Cogn. Sci. 12, 314-321.

Seth, A. K., Izhikevich, E., Reeke, G. N., and Edelman, G. M. (2006). Theories and measures of consciousness: an extended framework. Proc. Natl. Acad. Sci. U.S.A. 103, 10799-10804.

Soddu,A., Vanhaudenhuyse, A., Demertzi, A., Bruno, M.A., Tshibanda, L., Di, H., Boly, M., Laureys, S., and Noirhomme, Q. (2010). Resting state activity in patients with disorders of consciousness. Funct. Neurol. (in press).

Tononi, G. (2004). An information integration theory of consciousness. BMC
Neurosci. 5, 42. doi: 10.1186/14712202-5-42

Vanhaudenhuyse, A., Demertzi, A., Schabus, M., Noirhomme, Q., Bredart, S., Boly, M., Phillips, C., Soddu, A., Luxen, A., Moonen, G., and Laureys, S. (2010a). Two distinct neuronal networks mediate the awareness of environment and of self. J. Cogn. Neurosci. $23,570-578$.

Vanhaudenhuyse, A., Noirhomme, Q., Tshibanda, L. J., Bruno, M. A., Boveroux, P., Schnakers, C., Soddu, A., Perlbarg, V., Ledoux, D., Brichant, J. F., Moonen, G., Maquet, P., Greicius, M. D., Laureys, S., and Boly, M. (2010b). Default network connectivity reflects the level of consciousness in non-communicative brain-damaged patients. Brain 133, 161-171.

Vincent, J. L., Patel, G. H., Fox, M. D., Snyder, A. Z., Baker, J. T., Van Essen, D. C., Zempel, J. M., Snyder, L. H., Corbetta, M., and Raichle, M. E. (2007). Intrinsic functional architecture in the anaesthetized monkey brain. Nature 447, 83-86.

White, N. S., and Alkire, M. T. (2003). Impaired thalamocortical connectivity in humans during general-anestheticinduced unconsciousness. Neuroimage 19, 402-411.

Conflict of Interest Statement: The authors declare that the research was conducted in the absence of any commercial or financial relationships that could be construed as a potential conflict of interest.

Received: 11 June 2010; accepted: 07 December 2010; published online: 20 December 2010.

Citation: Noirhomme Q, Soddu A, Lehembre R, Vanhaudenhuyse A, Boveroux P, Boly M and Laureys S (2010) Brain connectivity in pathological and pharmacological coma. Front. Syst. Neurosci. 4:160. doi: 10.3389/fnsys.2010.00160

Copyright (C) 2010 Noirhomme, Soddu, Lehembre, Vanhaudenhuyse, Boveroux, Boly and Laureys. This is an open-access article subject to an exclusive license agreement between the authors and the Frontiers Research Foundation, which permits unrestricted use, distribution, and reproduction in any medium, provided the original authors and source are credited. 